\title{
Phase multistability of self-modulated oscillations
}

Sosnovtseva, Olga; Postnov, D.E.; Nekrasov, A.M.; Mosekilde, Erik; Holstein-Rathlou, N.H.

Published in:

Physical Review E. Statistical, Nonlinear, and Soft Matter Physics

Link to article, DOI:

10.1103/PhysRevE.66.036224

Publication date:

2002

Document Version

Publisher's PDF, also known as Version of record

Link back to DTU Orbit

Citation (APA):

Sosnovtseva, O., Postnov, D. E., Nekrasov, A. M., Mosekilde, E., \& Holstein-Rathlou, N. H. (2002). Phase multistability of self-modulated oscillations. Physical Review E. Statistical, Nonlinear, and Soft Matter Physics, 66(3), 036224. https://doi.org/10.1103/PhysRevE.66.036224

\section{General rights}

Copyright and moral rights for the publications made accessible in the public portal are retained by the authors and/or other copyright owners and it is a condition of accessing publications that users recognise and abide by the legal requirements associated with these rights.

- Users may download and print one copy of any publication from the public portal for the purpose of private study or research.

- You may not further distribute the material or use it for any profit-making activity or commercial gain

- You may freely distribute the URL identifying the publication in the public portal 


\title{
Phase multistability of self-modulated oscillations
}

\author{
O. V. Sosnovtseva, ${ }^{1,2}$ D. E. Postnov, ${ }^{1}$ A. M. Nekrasov, ${ }^{1}$ E. Mosekilde, ${ }^{2}$ and N.-H. Holstein-Rathlou ${ }^{3}$ \\ ${ }^{1}$ Physics Department, Saratov State University, Astrakhanskaya Street 83, Saratov, 410026, Russia \\ ${ }^{2}$ Department of Physics, The Technical University of Denmark, 2800 Kongens, Lyngby, Denmark \\ ${ }^{3}$ Department of Medical Physiology, Panum Institute, University of Copenhagen, 2200 Copenhagen N, Denmark
}

(Received 27 February 2002; published 27 September 2002)

\begin{abstract}
The paper examines the type of multistability that one can observe in the synchronization of two oscillators when the systems individually display self-modulation or other types of multicrest wave forms. The investigation is based on a phase reduction method and on the calculation of phase maps for vanishing and finite coupling strengths, respectively. Various phase-locked patterns are observed. In the presence of a frequency mismatch, the two-parameter bifurcation analysis reveals a set of synchronization regions inserted one into the other. Numerical examples using a generator with inertial nonlinearity and a biologically motivated model of nephron autoregulation are presented.
\end{abstract}

DOI: 10.1103/PhysRevE.66.036224

PACS number(s): 05.45.Xt

\section{INTRODUCTION}

Many processes in nature are characterized by a number of coexisting attractors that can be reached from different initial conditions but for a fixed set of parameters. This is observed in diverse areas of science, including physics $[1,2]$, chemistry $[3,4]$, and physiology $[5,6]$. In neuroscience, for instance, multistability is commonly considered a mechanism for memory storage and temporal pattern recognition [7]. Multistability phenomena have also been examined in systems with time delays [8] and noise-induced patterns [9]. In the present paper, we focus on phase multistability, i.e., the simultaneous existence of stable synchronous regimes with different phase relationships between the oscillations. This type of multistability was first observed for diffusively coupled oscillators that individually follow a perioddoubling route to chaos [10-13]. The possible synchronous regimes increase in number when more subharmonics of the basic frequency can be distinguished in the power spectrum. Phase multistability can also be observed for weak chaos that demonstrates an N-band structure. The hierarchy of multistability in systems of identical interacting oscillators with weak dissipative coupling has been studied numerically and experimentally by Astakhov et al. [10]. For two coupled Rössler systems, Rasmussen et al. [11] have found the replacement of some of the period-doubling bifurcations by torus birth bifurcations leading to quasiperiodicity, frequency locking, and the emergence of new nonsymmetric families of attractors. Anishchenko et al. have shown $[12,13]$ that this type of multistability is structurally stable with respect to a weak mismatch between the basic frequencies, and Postnov et al. [14] have described the nested structure of the phase synchronized regions.

Natural phenomena often involve dynamics with different time scales. This may be particularly significant for living systems. The thalamocortical relay neurons, for instance, can generate either spindle or delta oscillations [15]. Recently, Neiman and Russell [16] have found that the electroreceptors in paddlefish possess the property of being biperiodic. The functional units of the kidney, the nephrons, demonstrate low-frequency oscillations arising from a delay in the tubu- loglomerular feedback and somewhat faster oscillations associated with the inherent dynamics of the arteriolar radius [17]. Two-mode stochastic dynamics was studied in the context of rhythmic applause [18]. Likewise, in various engineering applications, special interest is paid to a fast dynamics subjected to a slow modulation or to complex behaviors characterized by brief bursts of oscillatory activity interspersed in quiescent periods [19].

As indicated by the above examples, the problem of synchronization of multimode oscillations is of significant interest. Yet, only a few studies seem to have considered this problem. Leaving the general aspect of synchronization of fast and slow motions for further investigations, in this paper we restrict ourselves to self-modulated oscillations that are widely spread in systems of different nature, leading, in its simplest form, to quasiperiodic behavior. Due to the internal coupling, the fast and slow oscillations will often be locked in some resonant ratio. The question then arises: How does the phase multistability manifest itself when systems demonstrating such resonant self-modulated behavior interact with each other? Below, we discuss some applicable methods and illustrate their use both for a three-dimensional model of an electronic oscillator and for a biologically motivated model of a nephron.

\section{APPROACH TO PHASE MULTISTABILITY}

The description of synchronization phenomena observed in interacting oscillators may be divided into two stages. The first step is to consider the case when the coupling strength is sufficiently weak so that an analytical method can be applied. The second step is to examine the case of finite coupling strength and show to what extent the results of the weak-coupling limit can be extrapolated. Since the definition of phase multistability involves the phase difference between the interacting oscillators, the phase variables will be the main quantities used to characterize the collective dynamics.

First, let us consider the weak-coupling case, i.e., we assume that the coupling causes only small perturbations of the limit cycles of the uncoupled oscillators. The coupled system may then be approximated by a phase model [20], where the 
phase $\phi$ of a limit cycle oscillator is defined by $d \phi\left(V_{0}\right) / d t$ $=1$ with $V_{0} \in R^{N}$ being a point on the limit cycle. Applying the concept of isochrons defined stroboscopically as a subset of initial conditions that asymptotically converge to the same point on the limit cycle [20], the phase description can be extended to some vicinity of the limit cycle. Moreover, for a sufficiently small vicinity of the limit cycle one can assume that the above subset is a flat surface that is transversal to the limit cycle in a given point.

In the presence of a small perturbation $P(V)$, the phase dynamics obeys the following equation [20]:

$$
\frac{d \phi}{d t}=1+Z(\phi) P(V)
$$

where the sensitivity function $Z(\phi)=\left.\operatorname{grad}_{V} \phi\right|_{V=V_{0}}$ measures the change of phase along the limit cycle caused by the change of $V$. Namely, we choose a point $V_{0}$ on the limit cycle and a point $V$ close to $V_{0}$ but not on the limit cycle and then measure the difference in phases between $V_{0}$ and $V$. In the limit $\left|V-V_{0}\right| \rightarrow 0$, this difference, divided by $\left|V-V_{0}\right|$, gives the sensitivity function $Z(\phi)$.

The interaction of two identical oscillators with phases $\phi_{1}$ and $\phi_{2}$ can be quantified by the evolution of their phase difference $\Delta \phi=\phi_{1}-\phi_{2}$. In the limit of weak interaction, averaged over a period, the phase dynamics for one of the oscillators can be expressed as [20]

$$
\frac{d(\Delta \phi)}{d t}=\Gamma(\Delta \phi)=\frac{1}{2 \pi} \int_{0}^{2 \pi} d \phi Z(\phi) P(\phi, \Delta \phi),
$$

where $P(\phi, \Delta \phi)=P\left(V_{0}(\phi), V_{0}(\phi+\Delta \phi)\right)$ describes the rate of change of the state vector $V$ of one oscillator due to the interaction with another oscillator with a phase difference $\Delta \phi$, and $Z P$ is the phase shift along the limit cycle for the given perturbation. Note, that the limit cycles in both systems are assumed to have similar shapes, i.e., to be topologically conjugated.

For mutually coupled oscillators, the entrainment manifests itself as a mutual phase shift. This can be analyzed purely in terms of the antisymmetric part $\Gamma_{a}(\Delta \phi)$ of the effective coupling function (2) [20]. The zeroes of $\Gamma_{a}(\Delta \phi)$ correspond to the phase-locked synchronous states $(\Delta \phi$ $=$ const $)$ and their stabilities are determined from the slope of $\Gamma_{a}(\Delta \phi)$ at the respective states, i.e., a negative slope means a stable state, and vice versa. This method of effective coupling has been used in a number of applications $[19,21,22]$.

When the coupling becomes strong enough to modify the geometry of the limit cycle, the phase reduction method can no longer be used. Direct numerical methods should then be applied. First of all, we calculate a set of points on the limit cycle modified by the interaction. Over a set of initial conditions covering the full length of the limit cycle, we follow the evolution of the initial phase shift $\Delta \phi(t)$ to some fixed value $\Delta \phi(t+\tau)$. Plotting these results together, i.e., $\Delta \phi(t$ $+\tau)$ vs $\Delta \phi(t)$, we obtain a one-dimensional phase map with a discrete time step $\tau$. The analysis of this map allows us to find the fixed points and estimate their stabilities.
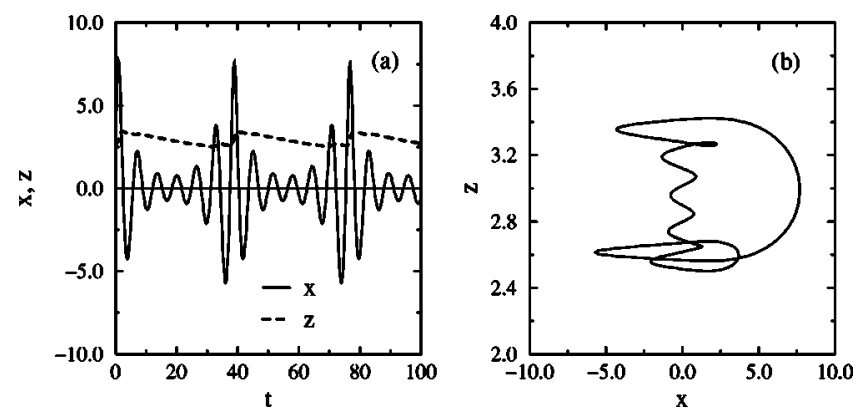

FIG. 1. Self-modulated regime $1: 6$ in a single generator with inertial nonlinearity. (a) Time series and (b) phase portrait $(m$ $=2.90328, g=0.012505$, and $b=5 \times 10^{-5}$ ).

Note that for the effective coupling method one can obtain the phase map in terms of $\Gamma_{a}$. Namely, for two coupled identical oscillators the phase difference behavior is given by [20]

$$
\frac{d(\Delta \phi)}{d t}=2 \Gamma_{a}(\Delta \phi)
$$

Setting $d t \rightarrow \tau$ and $d(\Delta \phi) \rightarrow\left(\Delta \phi_{t+\tau}-\Delta \phi_{t}\right)$ for small enough $\tau$ one finds the expression

$$
\Delta \phi_{t+\tau} \approx \Delta \phi_{t}+\tau 2 \Gamma_{a}\left(\Delta \phi_{t}\right),
$$

to which our numerical calculations converge for vanishing coupling.

\section{PHASE DYNAMICS OF COUPLED OSCILLATORS}

\section{A. Model equations and vector of diffusive coupling}

To illustrate our approach we use the three-dimensional model of an electronic oscillator (generator with inertial nonlinearity) that has a simple equivalent circuit implementation $[23,24]$ and a simple set of dynamical equations:

$$
\begin{gathered}
\dot{x}=m x-z x+y-b x^{3}, \\
\dot{y}=-x, \\
\dot{z}=-g z+g x(x+|x|) / 2 .
\end{gathered}
$$

Here, $m, b$, and $g$ are control parameters. With different values of these parameters, a variety of regular and chaotic regimes can be observed [24]. Among these, the model (5) can operate in a regime of self-modulated oscillations. This autonomous regime is characterized by slow and fast oscillatory modes whose frequencies are in a 1:6 ratio (Fig. 1).

In model systems, the coupling terms are generally considered to be proportional to the differences between the corresponding variables. For two coupled systems of the form (5), this implies the presence of terms of the form $\left(x_{1}\right.$ $\left.-x_{2}\right),\left(y_{1}-y_{2}\right)$, and $\left(z_{1}-z_{2}\right)$ in the equations for the $x, y$, and $z$ variables, respectively. The simplest case involves interaction through only one variable. Examples range from electronic circuits with a purely resistive coupling between the component circuits over mechanical oscillatory systems 

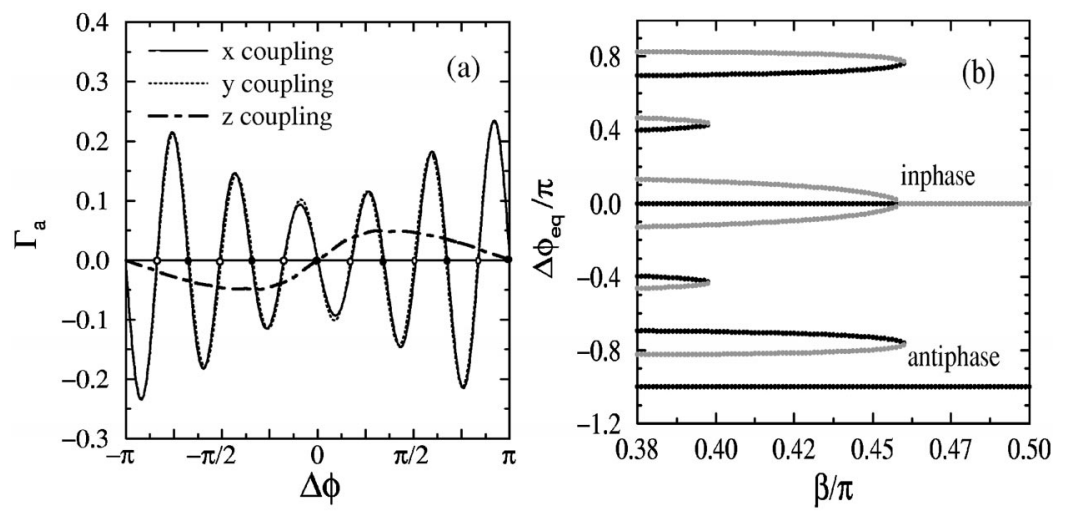

FIG. 2. Phase analysis for the self-modulated regime of a generator with inertial nonlinearity: (a) Antisymmetric part of effective coupling function; (b) evolution of location and stability of coexisting regimes when the coupling vector is gradually changed from $K_{x}$ to $K_{z}$. Black circles denote stable solutions. with inertial coupling to neuron models with electrical coupling. In more realistic circumstances, however, multivariable coupling seems to be more appropriate. For instance, the reactance in electronic circuits or the propagation time delay along neuronal axons may give rise to couplings through the velocity variable. Let us analyze the general case when the diffusive coupling is introduced in a vector form $\mathbf{K}$ $=\left(K_{x}, K_{y}, K_{z}\right)$

$$
\begin{gathered}
\frac{1}{\omega_{1,2}} \dot{x}_{1,2}=m x_{1,2}-z_{1,2} x_{1,2}+y_{1,2}-b x_{1,2}^{3}+K_{x}\left(x_{2,1}-x_{1,2}\right), \\
\frac{1}{\omega_{1,2}} \dot{y}_{1,2}=-x_{1,2}+K_{y}\left(y_{2,1}-y_{1,2}\right)
\end{gathered}
$$$$
\frac{1}{\omega_{1,2}} \dot{z}_{1,2}=-g z_{1,2}+g x_{1,2}\left(x_{1,2}+\left|x_{1,2}\right|\right) / 2+K_{z}\left(z_{2,1}-z_{1,2}\right),
$$

where $\omega_{1}=1$ and $\omega_{2}$ defines the frequency mismatch. It may be advantageous to represent the vector coupling in terms of polar coordinates:

$$
\begin{gathered}
K_{x}=K \cos \theta \cos \beta, \\
K_{y}=K \sin \theta \cos \beta, \\
K_{z}=K \sin \beta .
\end{gathered}
$$

This is the approach that we shall use in the following analysis. Here, $K$ denotes the coupling strength, and the angles 0 $\leqslant \theta \leqslant \pi / 2$ and $0 \leqslant \beta \leqslant \pi / 2$ define the relative weights of the three coupling terms. $\theta$ and $\beta$ can be also viewed as the orientation angles of the coupling force in the threedimensional subspace of each oscillator. Single-variable coupling is achieved when $(\theta=0, \beta=0),(\theta=\pi / 2, \beta=0)$, or $(\beta=\pi / 2)$.

\section{B. Application of phase reduction method}

To reach the regime of self-modulated oscillations for the system (5), we fix $m=2.90328, g=0.012505$, and $b$ $=0.0005$. Figure 2 illustrates the effect of phase multistability through the effective coupling technique. Inspection of the figure clearly shows that the calculated antisymmetric part of $\Gamma$ for $x$ and $y$ diagnose six stable and six unstable solutions. Note that their number corresponds to the number of local maxima over the period of oscillations (Fig. 1). Since the coupling is diffusive, the stable synchronous regimes in the coupled system are related to the coincidence of local maxima of oscillations in the individual units. The system is eventually stabilized in one of the stable regimes according to the considered initial conditions. The coupling has little influence on the phase difference of the system when the oscillator is in the synchronized regime. If any phase shift from this state arises, the system will gradually be attracted back to synchronous state.

Coupling via the $z$ variable demonstrates a completely different behavior. There is only one stable regime and this is in antiphase. We suppose that this is related to the dephasing effect $[21,22]$ caused by the vector field deformation in the vicinity of the saddle equilibrium point near the limit cycle. Variation of the $z$ variable strongly affects the distance of the perturbed trajectory from this point and, hence, is responsible for its slowing down or acceleration. Moreover, $z(t)$ operates in a different regime as compared to $x(t)$ and $y(t)$, i.e., without any modulation (Fig. 1). When the vector of diffusive coupling is changed from $x$ or $y$ coupling toward $z$ coupling, a transition between different sets of coexisting regimes can be observed. Figure 2(b) shows how the multistable regimes successively disappear with a smooth transition implemented by the variation of $\beta$ from $x$ to $z$ coupling.

\section{Mapping approach}

Let us consider the behavior of the coupled systems (6) for a strong interaction in order to compare the results with the case of vanishingly weak coupling.

As predicted by the phase reduction method, six phaselocked patterns for $K=0.0005$ are explicitly distinguished (Fig. 3). Each state corresponds to one of six stable equilibrium points in the phase map [Fig. 4(a)]. The time series of the multistable regimes are shifted with respect to each other while the phase portraits on the $\left(x_{1}, x_{2}\right)$ plane indicate different out-of-phase regimes with respect to the symmetric phase space.

When the mismatch parameter $\omega_{2}$ is varied away from the symmetric case the synchronous regimes sequentially lose their stability. The number of equilibrium points is decreased via tangent bifurcations in terms of the map [Fig. 4(b) with insert]. Figure 5 represents the bifurcation diagram of the 

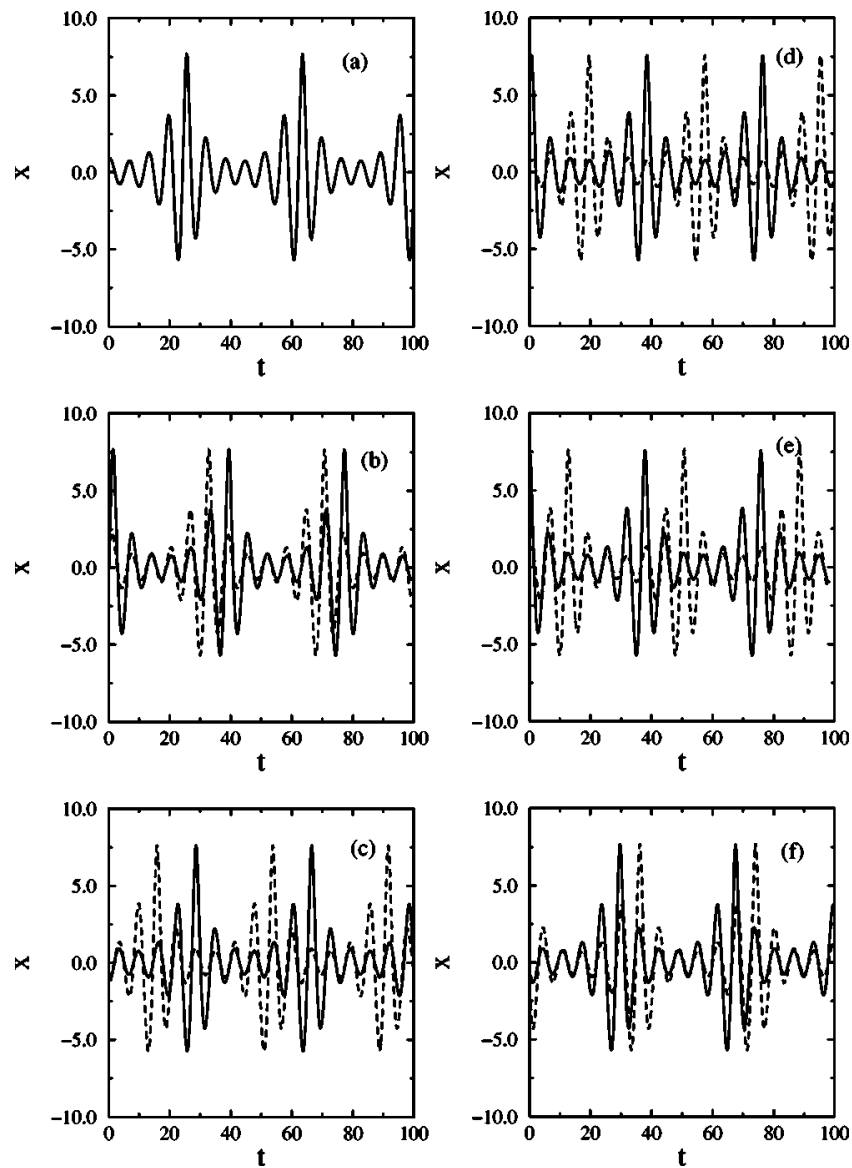

FIG. 3. Six phase-locked patterns with different phase shifts (a) $\Delta \phi=0.0, \quad$ (b) $\Delta \phi=1.6553 \pi, \quad$ (c) $\Delta \phi=1.3134 \pi$, (d) $\Delta \phi$ $=0.9928 \pi$, (e) $\Delta \phi=0.6710 \pi$, and (f) $\Delta \phi=0.3425 \pi$, when $K$ $=5 \times 10^{-4}$ and $\omega_{2}=1.0$.

possible synchronous regimes on the frequency mismatch vs coupling-strength parameter plane. For weak interaction, there are six stable (and the same number of unstable) solutions that differ from one another by a phase shift. There is a set of stability regions for different synchronous regimes whose structures are similar to those described in a previous publication [14] for oscillators demonstrating the Feigenbaum route to chaos. In present case, however, the tongues are not all inserted one into the other, but some of them are shifted a little with respect to each other. With increasing coupling, the solutions subsequently lose their stability through period-doubling bifurcations (dotted curves).

\section{ADJUSTMENT OF OSCILLATORY MODES IN NEPHRON AUTOREGULATION}

\section{A. Single-nephron model}

Over the years significant effort has been made to develop mathematical models that can describe the dynamical processes associated with the autoregulation of the functional unit of the kidney, the nephron. This regulation involves the the so-called tubuloglomerular feedback (TGF) mechanism by which the diameter of the afferent blood vessel is adjusted in response to the salt concentration in the fluid having the
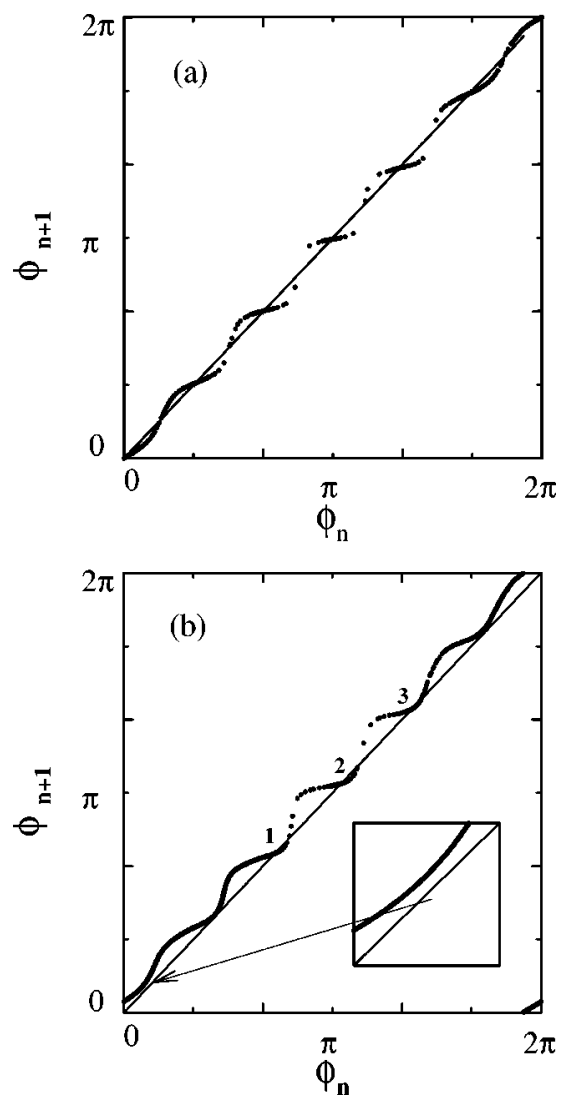

FIG. 4. The phase map of system (6) contains (a) six stable equilibrium points corresponding to six synchronous regimes for identical systems $\left(\omega_{2}=1.0\right)$. When a frequency mismatch $\left(\omega_{2}\right.$ $=1.001$ ) is introduced (b) only three equilibrium points remain. $K$ is fixed at $5 \times 10^{-4}$.

loop of Henle [25-28]. A particular aspect of this research has been to show that the transition from regular tubular pressure oscillations (as observed in rats with normal blood pressure) to irregular variations (as observed in hypertensive rats) can be explained in terms of parameter changes within the framework of well-established physiological mechanisms.

The autoregulation in an individual nephron may be described by the following model $[28,29]$ :

$$
\begin{gathered}
\dot{P}_{t}=\frac{1}{C_{t u b}}\left\{F_{f}\left(P_{t}, r\right)-F_{r e a b}-\left(P_{t}-P_{d}\right) / R_{H}\right\}, \\
\dot{r}=v_{r}, \\
\dot{v}_{r}=\frac{1}{\omega}\left\{P_{a v}\left(P_{t}, r\right)-P_{e q}\left[r, \Psi\left(X_{3}, \alpha\right), T\right]-\omega d v_{r}\right\},
\end{gathered}
$$

$$
\begin{gathered}
\dot{X}_{1}=\frac{1}{R_{H}}\left(P_{t}-P_{d}\right)-\frac{3}{T} X_{1}, \\
\dot{X}_{2}=\frac{3}{T}\left(X_{1}-X_{2}\right),
\end{gathered}
$$




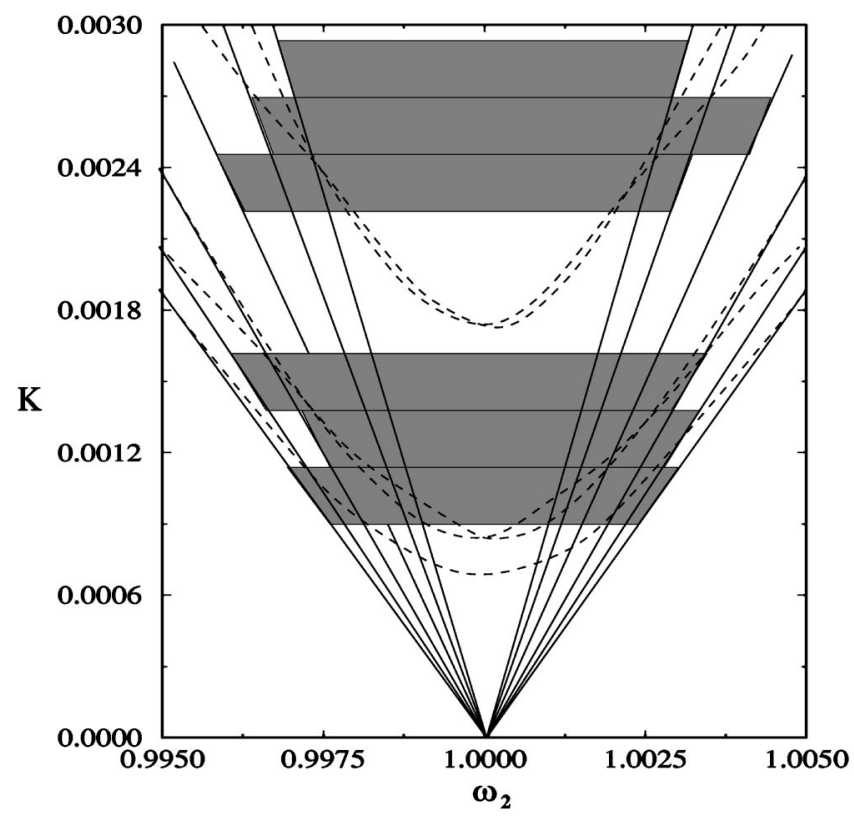

FIG. 5. Synchronization regions for coexisting families of attractors $\left(m=2.90328, g=0.012505\right.$, and $\left.b=5 \times 10^{-5}\right)$. Dotted curves denote period-doubling bifurcations.

$$
\dot{X}_{3}=\frac{3}{T}\left(X_{2}-X_{3}\right)
$$

The first equation represents the pressure variations in the proximal tubule in terms of the in and outgoing fluid flows. Here, $F_{f}$ is the single-nephron glomerular filtration rate, and $C_{t u b}$ is the elastic compliance of the tubule. The flow into the loop of Henle is determined by the difference $\left(P_{t}-P_{d}\right)$ between the proximal and the distal tubular pressures and by the flow resistance $R_{H}$. The reabsorption in the proximal tubule $F_{\text {reab }}$ is assumed to be constant.

The following two equations describe the dynamics associated with the flow control in the afferent arteriole. Here, $r$ represents the radius of the active part of the vessel and $v_{r}$ is its rate of increase. $d$ denotes a characteristic time constant describing the damping of the oscillations. $\omega$ is a measure of the mass relative to the elastic compliance of the arteriolar wall, $P_{a v}$ denotes the average pressure in the active part of the arteriole, and $P_{e q}$ is the value of this pressure for which the arteriole is in equilibrium with its present radius and muscular activation. The expressions for $F_{f}, P_{a v}$, and $P_{e q}$ involve a number of algebraic equations that must be solved along with the integration of Eq. (8).

The remaining equations in the single-nephron model describe the delay $T$ in the TGF regulation. This delay arises both from the transit time through the loop of Henle and from the cascaded enzymatic processes between the macula densa cells and the smooth muscle cells that control the contractions of the afferent arteriole.

The feedback delay $T$, that typically assumes a value of 12-18 s, will be considered the main bifurcation parameter in our analysis. Another important parameter is the strength $\alpha$ of the feedback regulation. This parameter takes a value about 12 for normotensive rats and increases to about 18 for
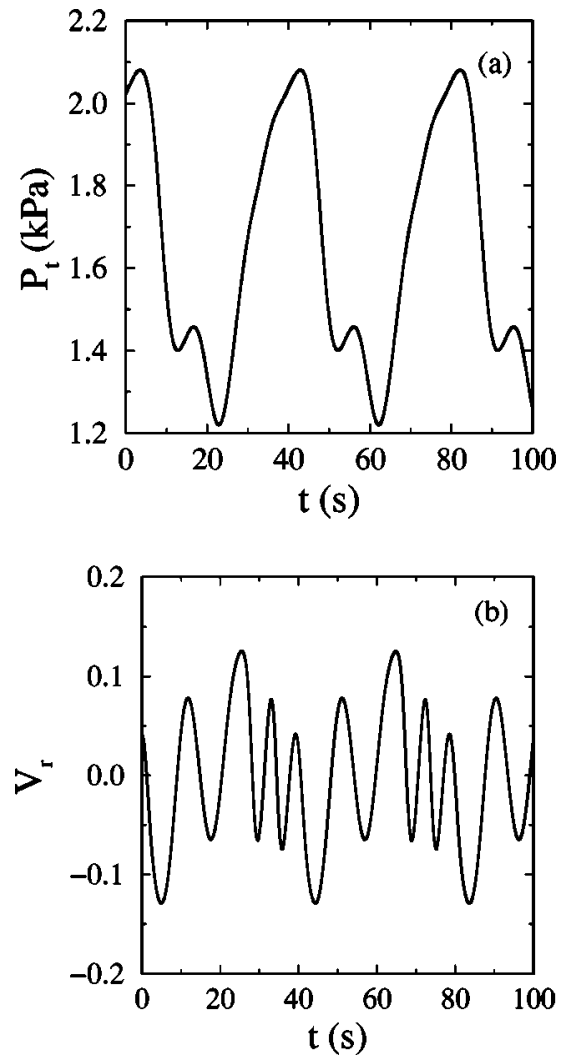

FIG. 6. (a) Variation of the tubular pressure $P_{t}$ and (b) the rate of change $v_{r}$ for the arteriolar radius in the self-modulated 1:4 regime.

hypertensive rats [30]. For a more detailed explanation of the model equations, control parameters, and the dynamics of nephrons see Refs. [28,29,31].

Postnov et al. [17] recently examined the interaction between the two oscillatory modes in the single nephron model. These modes can adjust their dynamics so as to attain different states with rational relations $(1: n)$ between the periods. The main $(1: 1)$ synchronization regime is located near $T=2 \mathrm{~s}$, but regions of higher resonances $(1: 4,1: 5$, and 1:6) exist in the physiologically interesting range for the delay time $T \in[12 \mathrm{~s}, 20 \mathrm{~s}]$. As an illustration, Fig. 6 shows an example of 1:4 oscillations. Among the resonance regions there are regions where the modes fail to synchronize, and quasiperiodic or chaotic dynamics result. Thus, an individual nephron can operate in a self-modulated regime, providing a realistic example for our study of phase multistability. In the kidney, nephrons are often arranged in pairs with a common piece of afferent arteriole and the interaction is realized in a complex way. There are at least two different mechanisms of mutual coupling, the hemodynamic coupling and the socalled vascular propagated coupling. The first originates from a simple redistribution of the incoming blood flows in response to the contraction of one of the arterioles, and the second is associated with electrochemical signals that propagate along the arteriolar walls. Thus, it is not easy to determine the origin of the complex behavior of paired nephrons. To achieve a better understanding of this problem, let us first test the individual nephron model with weak diffusive all- 


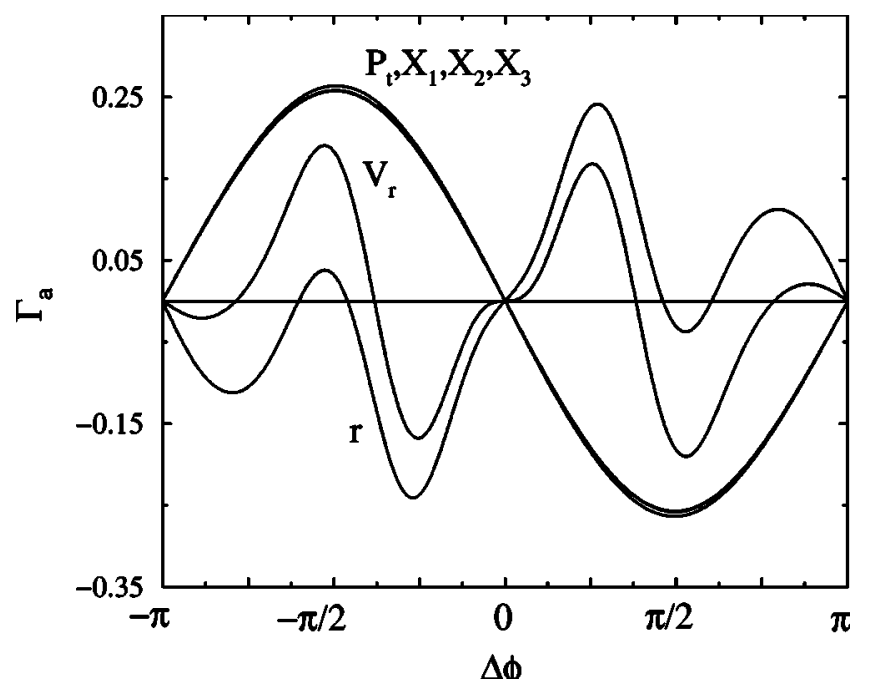

FIG. 7. Antisymmetric part of the effective coupling function for the coupled nephrons (8) at $T=16.0 \mathrm{~s}$ and $\alpha=18.6$. The behavior of $\Gamma_{a}(\Delta \phi)$ reveals the synchronization properties of two coupled 6-dimensional oscillators.

variable coupling. Using the effective coupling approach, we will estimate the specific properties of model (8) and then analyze the phase dynamics of the coupled nephrons in more realistic circumstances.

\section{B. Weak diffusive coupling}

Suppose that two models of the form (8) are coupled via the vector diffusive coupling [see model (6)]. Figure 7 illustrates the antisymmetric parts $\Gamma_{a}$ of the effective coupling function calculated separately for the six cases of onevariable coupling. For the variables $P_{t}, X_{1}, X_{2}$, and $X_{3}$ the plots of $\Gamma_{a}$ almost coincide and possess a zero point with negative slope at $\Delta \phi=0$. This means that a single in-phase synchronous regime can be found when two nephron models are diffusively coupled through these variables. It is also clearly seen that the synchronous antiphase regime is unstable $(\Delta \phi=\pi)$. Generally, the observed behavior is very natural and expected for a wide class of classical oscillators (such as Van der Pol oscillators). Phase multistability can not be detected.

However, a coupling via the $r$ or $v_{r}$ variables reveals a more complex behavior. The $\Gamma_{a}$ curve for $r$ has three stable and three unstable equilibrium points. Note that the in-phase regime $\Delta \phi=0$ is unstable. Similar behavior is observed for $v_{r}$, but in this case the in-phase regime is related to a "neutral" equilibrium point, $d\left(\Gamma_{a}\right) /\left.d(\Delta \phi) \rightarrow 0\right|_{\Delta \phi \rightarrow 0}$.

Thus, application of the phase reduction method to the relatively high-dimensional nephron model allows us to diagnose a number of qualitatively different responses of this oscillator to varying coupling components. This is in good agreement with both experimental data and simulation results. It is known that the $P_{t}, X_{1}, X_{2}, X_{3}$, and $v_{r}$ variables are involved in the tubuloglomerular feedback loop that is responsible for low-frequency oscillations with a period $T_{h}$ $=2.2 T$ (as before, $T$ denotes the delay in the TGF regulation). On the other hand, the $r$ and $v_{r}$ variables, demonstrat- ing somewhat faster oscillations with a period $T_{v} \approx T_{h} / 4$, are associated with the adjustment of the arteriolar radius. Hence, different nephron responses to distinct types of coupling are related to the above biological processes.

Let us make an interesting observation. With a 1:4 ratio between the frequencies of the oscillatory modes, one can expect four coexisting stable regimes for the coupling via $r$ or $v_{r}$, as it was observed for the electronic oscillator. However, only three such regimes (zero points with negative slope) can be found in Fig. 7. We believe that this result is due the fact that the oscillatory modes in the single nephron are not actually weakly coupled. Thus, the properties of the point $\Delta \phi=0$ are defined through the competition of oscillatory modes that can compensate each other even when the coupling takes place via one variable. To get an additional stable point, coupling via the $P_{t}$ variable should be introduced. This is in a good agreement with our biological understanding of the model.

\section{Biologically motivated coupling}

As discussed in Ref. [29], neighboring nephrons can influence each other's blood supply either through vascularly propagated electrical (or electrochemical) signals or through a direct hemodynamic coupling arising via the redistribution of the blood flow to the coupled nephrons. While the hemodynamic coupling depends mainly on the flow resistances in the arteriolar network, the vascularly propagated coupling is associated with a characteristic propagation length of the order of $l=200 \mu \mathrm{m}$. The result is that, only nephrons situated close to one another can interact via the vascularly propagated coupling [32,33]. Nephrons situated further apart but sharing a common piece of interlobular artery may interact via the hemodynamic coupling. In our mathematical model [29], the two interaction mechanisms are included via nonlinear functions for the hemodynamic coupling with a strength $\varepsilon$ and for the vascularly propagated coupling with a strength $\gamma$.

In reality, we expect both mechanisms to be present simultaneously and to compete for dominance. Depending on the precise structure of the arteriolar network this may cause one mechanism to be the stronger in certain parts of the kidney and the other mechanism to dominate in other parts. Pure vascularly propagated or pure hemodynamic coupling are assumed to cause in-phase and antiphase entrainment, respectively. But their combination give rise to a set of coexisting regimes whose origin and evolution is in a good agreement with findings for diffusively coupled selfmodulated oscillators as described in Sec. III.

The coupling between nephrons leads to a set of coexisting self-sustained oscillations that are characterized by the different phase relations for the fast and slow motions. These oscillations coexist at the same range of parameters but for different initial conditions. Since the single nephron operates in the 1:4 self-modulated regime, four phase shifted solutions may coexist in the coupled system (Fig. 8).

Figure 9 shows a segment of the bifurcation diagram for synchronous solutions on the parameter mismatch vs hemodynamic-coupling parameter plane. Here, the mismatch 

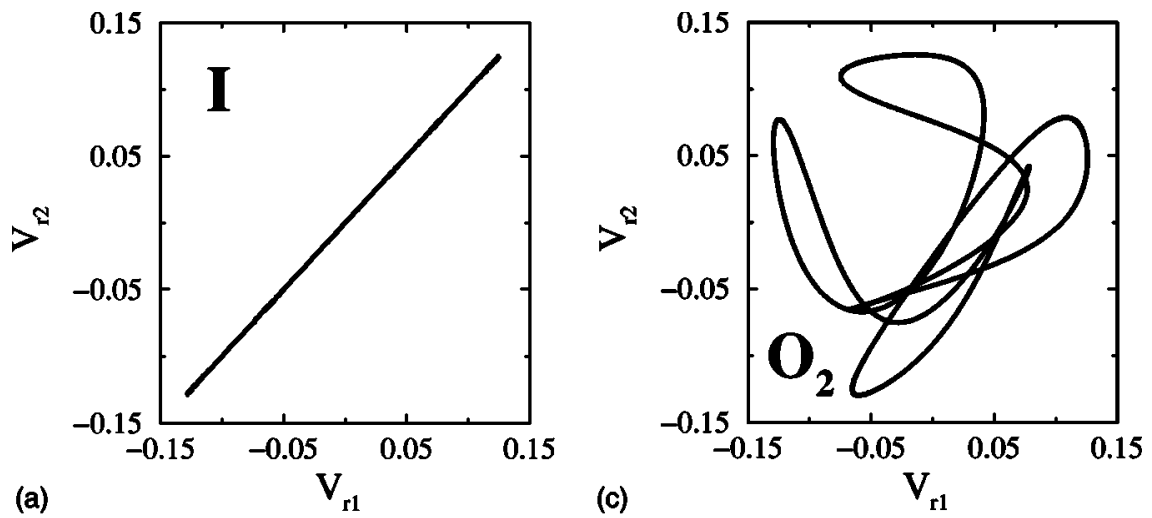

FIG. 8. Phase portraits for different regimes. In-phase $(\Delta \phi$ $=0.0)$ and antiphase $(\Delta \phi$ $=1.5711 \pi)$ solutions are labeled $I$ and $A$, respectively. Two out-ofphase solutions with $\Delta \phi$ $=1.7526 \pi$ and $\Delta \phi=0.9272 \pi$ are indicated as $\mathrm{O}_{1}$ and $\mathrm{O}_{2}$. The phase space trajectories are projected onto the planes spanned by the rates of change for the two arteriolar radii.
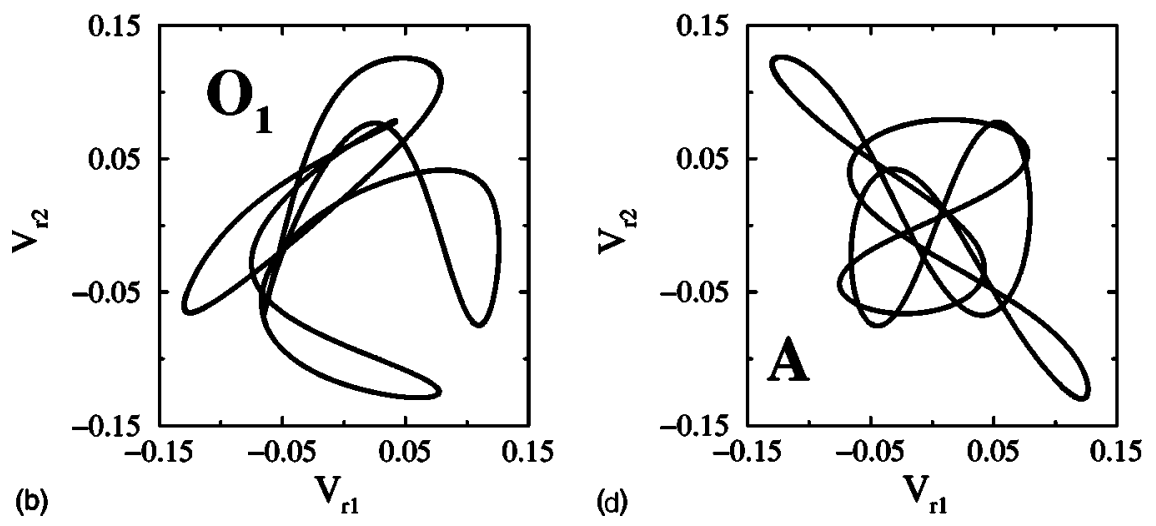

parameter is taken to be the ratio of the delay times for the two nephrons. The strength of the vasculary propagated interaction is fixed at $\gamma=0.004$. In-phase oscillations are stable when both interacting systems are nearly identical $\left(T_{1}\right.$ $\approx T_{2}$ ) and the hemodynamic coupling is weak enough ( $\varepsilon$ $<0.0115)$. However, due to the self-modulated nature of the oscillations in the individual nephron, there are also two outof-phase stable synchronous regimes $\left(O_{1}\right.$ and $\left.O_{2}\right)$. Their

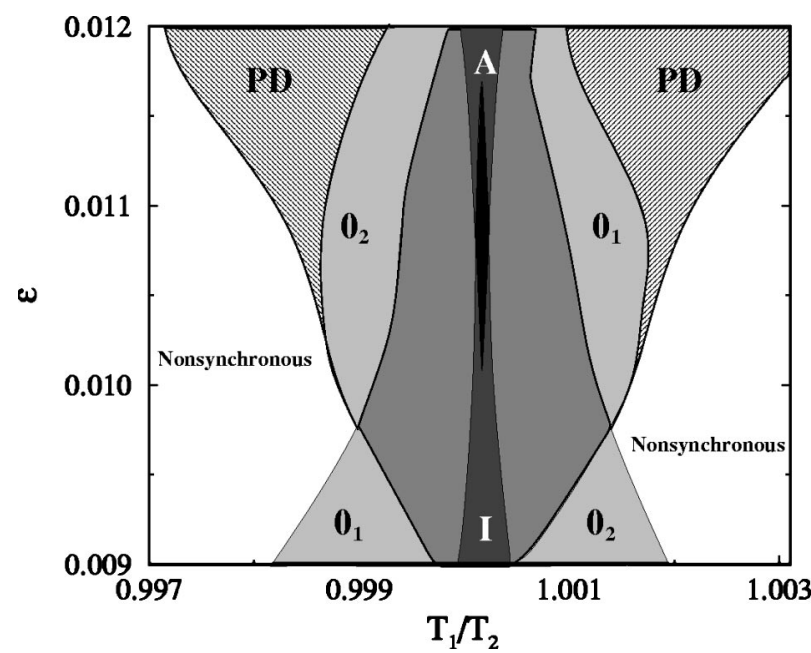

FIG. 9. Synchronization regions for coexisting families of attractors $\left(\alpha=18.595, T_{2}=16.0 \mathrm{~s}, \quad \gamma=0.004\right) . I$ denotes the stable in-phase solution, $A$ the antiphase solution, and $O_{1}$ and $O_{2}$ are two out-of-phase solutions. PD denotes regimes with period-doubled solutions.
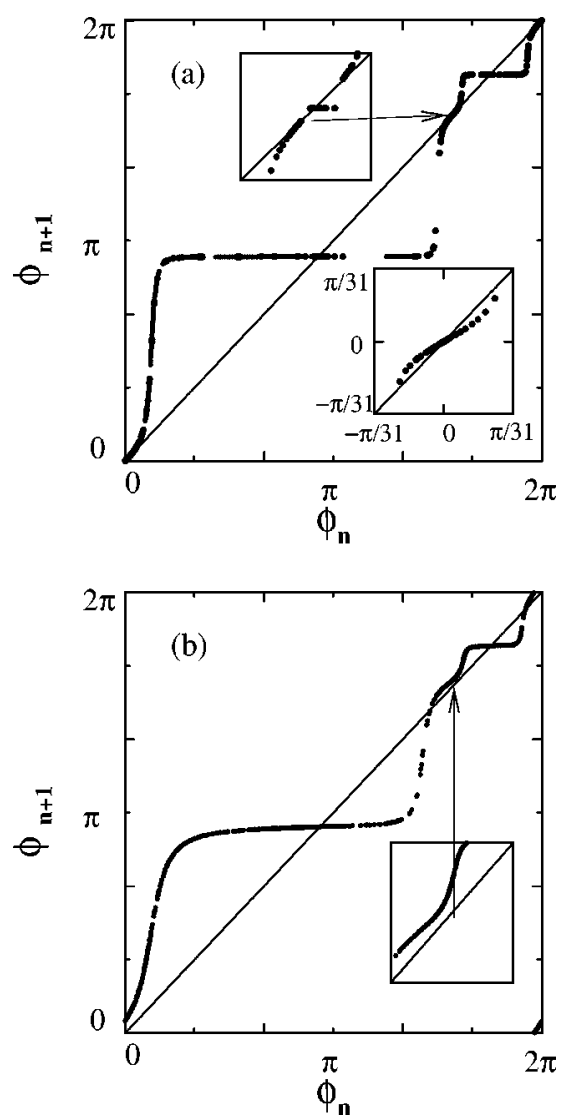

FIG. 10. Phase map demonstrates (a) four stable equilibrium points for identical systems $\left(T_{1}=T_{2}=16.0 \mathrm{~s}\right)$ and (b) two stable solutions when a mismatch is introduced $\left(T_{1}=15.9985 \mathrm{~s}, T_{2}\right.$ $=16.0 \mathrm{~s}) .(\alpha=18.595, \varepsilon=0.01093$, and $\gamma=0.004$. $)$ 
existence follows directly from the above results on weak diffusive coupling via $v_{r}$.

When $\varepsilon$ increases, the antiphase regime $A$ also becomes stable due to the effect of the hemodynamic coupling. Within some interval of $\varepsilon$, there are four stable coexisting solutions: in-phase $I$, antiphase $A$, and two out-of-phase regimes $O_{1}$ and $O_{2}$. The phase map for $\gamma=0.004, T_{1}=T_{2}=16.0 \mathrm{~s}$, and $\varepsilon=0.010930$ is given in Fig. 10(a). Eight fixed points can be detected, four of them are stable and correspond to the above discussed synchronous regimes.

When a mismatch $T_{1} \neq T_{2}$ is introduced, these regimes sequentially lose their stability. In accordance with the phase map, the in-phase or antiphase regimes disappear through a tangent bifurcation. An example, when only two stable fixed point remain, is given in Fig. 10(b). With increasing mismatch, the $O_{1}$ and $O_{2}$ cycles also loses their stability via a tangent bifurcation (entering the nonsynchronous region) or via a period doubling at the border of the $P D$ zone in Fig. 9.

Thus, we have observed that even under the conditions of a complex two-channel coupling, the interacting oscillatiors in the self-modulated regime can preserve the main features with respect to phase multistability. Namely, for the 1:4 regime of the individual nephron there are four synchronous regimes with different phase shifts in the 1:1 internephron synchronization region.

\section{SUMMARY}

The results of our numerical simulations can be summarized as follows. A system of two diffusively coupled oscillators operating in the $1: n$ regime of self-modulation ( $n$ being an integer) reveals the same aspects of phase multistability as previously discussed for systems with period-doubling cascades [14].
For coupled identical oscillators one can expect $n$ coexisting synchronous solutions that differ from one another by phase shifts. The corresponding synchronization region consists of a set of Arnol'd tongues embedded one into the other or shifted with respect to each other. The evolution of multistable states depends significantly on the vector of diffusive coupling, i.e., on the relation of coupling strength for different equations. Hence, one can use the presence of particular states of synchronization in experimental results as a signature of the interaction mechanism. This is of significant interest in biological and physiological studies where one may not always know the coupling mechanisms from other analyses.

Our results on phase multistability of self-modulated regimes have been tested for a high-dimensional model of interacting nephrons. The analysis reveals different responses depending on which time scale (fast or slow) the interaction influences. We have also found that phase multistability takes place in a realistic model of two-channel coupled nephrons and strongly depends on the relations between the strength of the two interaction mechanisms.

We believe that the multistability analysis of phase-locked patterns in systems with fast dynamics subjected slow modulation can provide a better understanding of many regulation and adaptation processes in nature.

\section{ACKNOWLEDGMENTS}

This work was partly supported by INTAS Grant No. (012061) and RFBR (01-02-16709). O.S. aknowledges the Natural Science Foundation of Denmark and INTAS (Grant No. YSF 01/1-0023).
[1] M. Brambilla, L. A. Lugiato, V. Penna, F. Prati, C. Tamm, and C. O. Weiss, Phys. Rev. A 43, 5114 (1991).

[2] N. G. Sun and G. P. Tsironis, Phys. Rev. B 51, 11221 (1995).

[3] K. L. C. Hunt, J. Kottalam, M. D. Hatlee, and J. Ross, J. Chem. Phys. 96, 7019 (1992).

[4] R. S. MacKay and J. A. Sepulchre, Physica D 82, 243 (1995).

[5] C. C. Canavier, D. A. Baxter, J. W. Clark, and J. H. Byrne, J. Neurophysiol. 69, 2252 (1993).

[6] J. Foss, A. Longtin, B. Mensour, and J. Milton, Phys. Rev. Lett. 76, 708 (1996).

[7] J. Hertz, A. Krogh, and R. G. Palmer, Introduction to the Theory of Neural Computation (Addision-Wesley, New York, 1991).

[8] S. Kim, S. H. Park, and C. S. Ryu, Phys. Rev. Lett. 79, 2911 (1997).

[9] S. Kim, S. H. Park, and C. S. Ryu, Phys. Rev. Lett. 78, 1616 (1997).

[10] V. V. Astakhov, B. P. Bezruchko, E. N. Erastova, and E. P. Seleznev, Zh. Tekh. Fiz. 60, 19 (1990) [Sov. Phys. Tech. Phys. 35, 1122 (1990)].

[11] J. Rasmussen, E. Mosekilde, and Ch. Reick, Math. Comput. Simul. 40, 247 (1996).
[12] V. S. Anishchenko, V. V. Astakhov, T. E. Vadivasova, O. V. Sosnovtseva, and L. O. Chua, Int. J. Bifurcation Chaos Appl. Sci. Eng. 5, 1677 (1995).

[13] T. E. Vadivasova, O. V. Sosnovtseva, A. G. Balanov, and V. V. Astakhov, Discrete Dyn. Nat. Soc. 4, 231 (2000).

[14] D. E. Postnov, T. E. Vadivasova, O. V. Sosnovtseva, A. G. Balanov, and E. Mosekilde, Chaos 9, 227 (1999).

[15] X.-J. Wang, Neuroscience 59, 21 (1994).

[16] A. Neiman and D. F. Russell, Phys. Rev. Lett. 86, 3443 (2001).

[17] D. E. Postnov, O. V. Sosnovtseva, E. Mosekilde, and N.-H. Holstein-Rathlou, Int. J. Mod. Phys. B 15, 3079 (2001).

[18] A. Nikitin, Z. Néda, and T. Vicsek, Phys. Rev. Lett. 87, 024101 (2001).

[19] S. H. Park, S. Kim, H.-B. Pyo, and S. Lee, Phys. Rev. E 60, 2177 (1999).

[20] Y. Kuramoto, Chemical Oscillations, Waves, and Turbulence (Springer-Verlag, New York, 1984).

[21] S. K. Han, C. Kurrer, and Y. Kuramoto, Phys. Rev. Lett. 75, 3190 (1995).

[22] D. Postnov, S. K. Han, and H. Kook, Phys. Rev. E 60, 2799 (1999). 
[23] V. S. Anishchenko, T. E. Vadivasova, D. E. Postnov, and M. A. Safonova, Int. J. Bifurcation Chaos Appl. Sci. Eng. 2, 633 (1992).

[24] V. S. Anishchenko, Dynamical Chaos-Models and Experiments: Appearance Routes and Structure of Chaos in Simple Dynamical Systems (World Scientific, Singapore, 1995).

[25] K. S. Jensen, E. Mosekilde, and N.-H. Holstein-Rathlou, Mondes en Developement 54/55, 91 (1986).

[26] N.-H. Holstein-Rathlou and D. J. Marsh, Am. J. Physiol. 258, F1448 (1990).

[27] N.-H. Holstein-Rathlou and D. J. Marsh, Bull. Math. Biol. 56, 441 (1994).
[28] M. Barfred, E. Mosekilde, and N.-H. Holstein-Rathlou, Chaos 6, 280 (1996).

[29] E. Mosekilde, Topics in Nonlinear Dynamics: Applications to Physics, Biology and Economic Systems (World Scientific, Singapore, 1996).

[30] P. P. Leyssac and N.-H. Holstein-Rathlou, Pflügers Arch. 413, 267 (1989).

[31] N.-H. Holstein-Rathlou, K.-P. Yip, O. V. Sosnovtseva, and E. Mosekilde, Chaos 11, 417 (2001).

[32] N.-H. Holstein-Rathlou, Pflügers Arch. 408, 438 (1987).

[33] A. J. Wagner, N.-H. Holstein-Rathlou, and D. J. Marsh, Am. J. Physiol. 274, F372 (1997). 